\title{
High performance membrane materials for gas separation
}

\author{
Aleksandra Wolińska-Grabczyk ${ }^{1}$, Andrzej Jankowski \\ Centre of Polymer and Carbon Materials, Polish Academy of Sciences, \\ M. Curie-Skłodowskiej 34, 41-819 Zabrze, Poland
}

\begin{abstract}
The use of membranes in various gas separations has increased significantly in recent times. This review presents some of the recent noteworthy advances in the field of membranes materials for these applications. A description of the most promising groups of high free volume polymers, including polyimides, thermally rearranged (TR) polymers, substituted polyacetylenes, perfluoropolymers, and polymers with intrinsic microporosity (PIM) is provided. High performance, rubbery, polyethers and polyether based copolymers are shown as another important class of polymer membrane materials. The development of inorganic membranes, which are not bound to the trade-off limitations between permeability and selectivity exhibited by polymers is also presented. The attention is focused on zeolitic materials, metal organic frameworks (MOF), and carbon molecular sieves (CMS). The field of mixed matrix membranes composed of inorganic particles embedded in a polymer matrix is also briefly outlined.
\end{abstract}

Keywords: Polymeric membranes; Inorganic membranes; Mixed matrix membranes; Gas separation

Gas separation using membrane technology has gained increasing economic importance as a viable energy saving alternative to other conventional separation techniques. Commercial gas separation membranes are made of synthetic polymer materials. These materials offer a range of desired properties such as low cost, easy processability, good permeability, and good mechanical stability. Inorganic membranes exhibit the advantage of higher permeabilities and selectivities, as well as much higher resistance to harsh chemical conditions, high temperature and pressure. However, their immediate application is still hindered by the lack of technology allowing formation of defect free membranes, by the high cost of membrane production, and inherent brittleness causing handling problems. A new approach has emerged recently providing an alternative membrane structure called mixed matrix membrane. This membrane is composed of inorganic particles embedded in the polymer matrix and have the potential of achieving better separation performance due to the inner separation characteristics of the inorganic material and better processability as a result of the flexibility of the polymer matrix. This work is focused on the recent advances in those three groups of membrane materials.

\section{Polymeric membrane materials}

Over the last 30 years, several polymers have been established as common gas separation membranes. They all belong to glassy polymers, which are more selective though less permeable than rubbers. The commercially relevant polymers include polysulphones, polycarbonates, cellulose acetates, poly(phenylene oxides), aramids, and polyimides.

\footnotetext{
${ }^{1}$ Corresponding author: (A.Wolińska-Grabczyk) awolinska@cmpw-pan.edu.pl
} 
To increase membrane performance, several modifications of the polymer structure have been introduced. However, the obtained structure-property data showed that a trade-off between permeability and selectivity exists, which does not allow for the improvement of the one parameter without negatively affecting the another one. This trade-off became apparent when log-log plots of selectivity versus permeability of the more permeable gas were demonstrated by Robeson in 1991 [1]. It was observed that all the experimental data points utilized to establish the correlation exist below a well- defined line called "upper-bound trade-off" relationship. The upper-bound relationship was shown to be valid for a multitude of gas pairs.

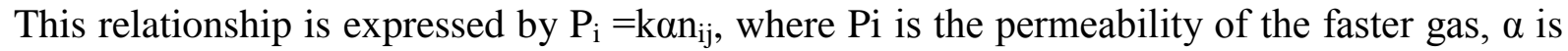
the separation factor $\left(\mathrm{P}_{\mathrm{i}} / \mathrm{P}_{\mathrm{j}}\right)$, and $\mathrm{n}$ is the slope of the upper-bound line, and $\mathrm{k}$ is a constant for the given gas pair. An example of this relationship for $\mathrm{CO}_{2} / \mathrm{N}_{2}$ gas pair is given in Fig.1.

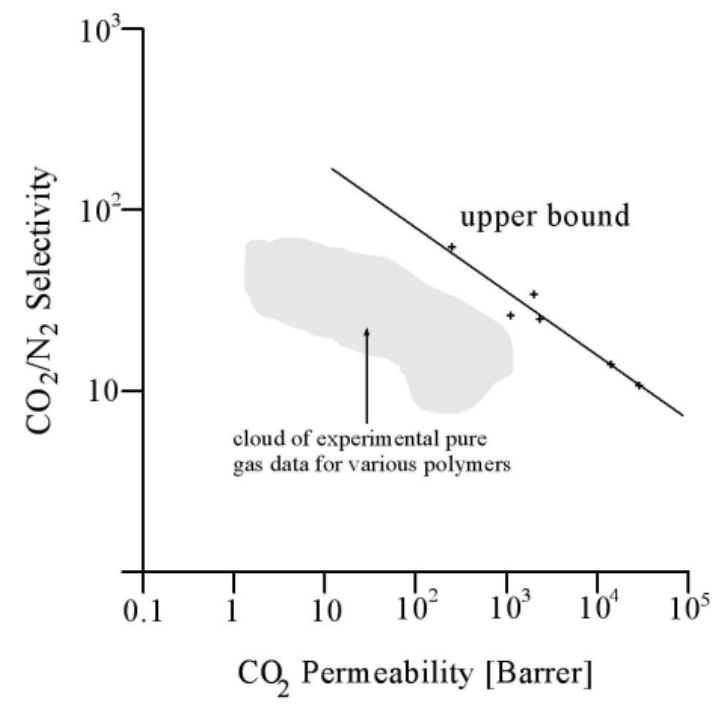

Fig.1. The upper bound correlation for $\mathrm{CO}_{2} / \mathrm{N}_{2}$ separation; based on the data from [1]

To overcome the trade-off relation several strategies have been adopted. Along with continued modification of the existing groups of polymers, new classes of membrane materials have also emerged recently. The most promising groups of those materials will be briefly characterized. They all belong to the type of high free volume glassy polymers. Their chemical structures are given in Fig.2.

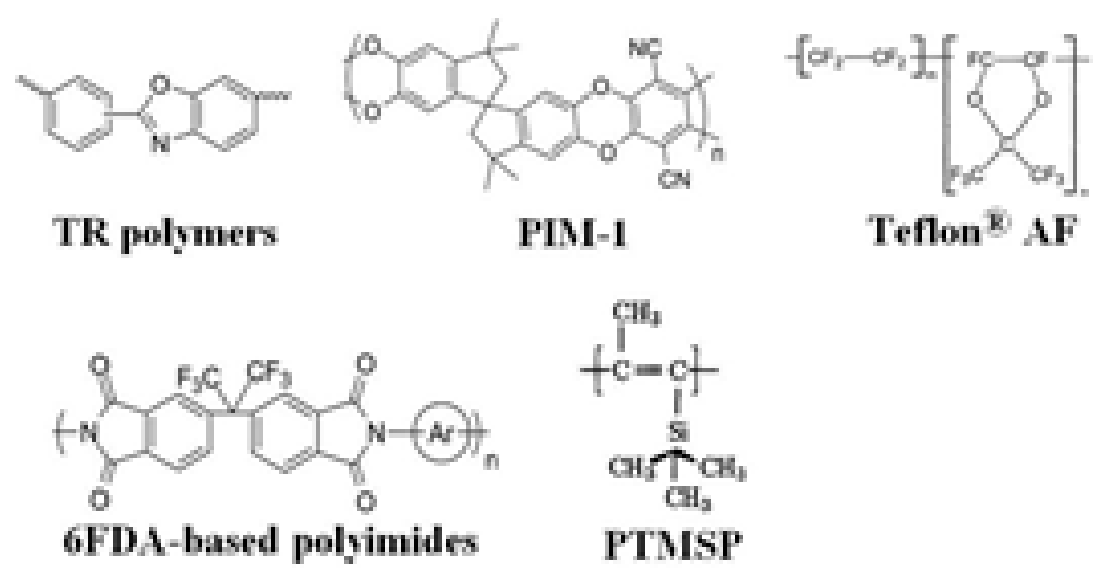

Fig.2 Chemical structures of selected high free volume polymers. 
Polyimides have been one of the most extensively investigated polymer materials for gas separations. Many of them exhibit very high selectivity but generally modest permeability. However, it appeared that by using proper diamines and dianhydrides, their properties can be successfully tailored leading to polymers with improved both permeability and selectivity. Particularly, the polyimides containing hexafluoro groups in the dianhydride moiety and bulky substituents in the diamine unit have made up a new generation of polyimides, which are placed close to the Robeson $\mathrm{CO}_{2} / \mathrm{CH}_{4}$ upper bound. The most permeable and highly soluble polyimides reported up to now are those synthesized using dianhydride with a spirocentre. Aromatic polyimides containing $-\mathrm{OH}$ (or $-\mathrm{SH}$ ) groups in orto position to the imide ring are also precursors of a new family of polymeric membrane materials termed thermally rearranged polymers (TR).

Thermally rearranged polymers, which have polybenzoxazole (PBO) (or polybenzotiazole) structure, are formed by a molecular thermal rearrangement of a respective polyimide at high temperatures $\left(350-450^{\circ} \mathrm{C}\right)$ in an inert atmosphere. They have an excellent separation performance, well above Robeson's upper bound for the $\mathrm{CO}_{2} / \mathrm{CH}_{4}$ gas pair. In addition to the high both permeability and selectivity, those polymers show also high resistance to the $\mathrm{CO}_{2}$ induced plasticization. The TR concept has also been applied to structurally different copolymer precursors leading to membrane materials which exhibited various transport properties depending on the ratio of PBO to polyimide units. The outstanding permeation and separation characteristics of TR polymers have been attributed to an enhanced fractional free volume and narrowed free volume distribution.

Substituted polyacetylenes constitute another class of highly permeable polymers. Their high permeability is resulting from the presence of bulky substituents attached to the main chain. These substituents inhibit conformational change and make the polymer backbone to adapt a twisted shape. As a result, polyacetylenes, when in the solid state, exhibit high free volume which comprises smaller disconnected free volume elements, such as those present in typical glassy polymers, and large continuous microvoids. The large free volume allows for high gas solubility, whereas microvoids for high diffusion coefficients, both leading to exceptionally high permeability. Poly(trimethylsilyl propyne), PTMSP, the most known member of this group, shows oxygen permeability ten times higher than that of the rubbery poly(dimethylsiloxane) (PDMS). However, the high permeability of the substituted polyacetylenes comes at the expense of a rather low selectivity. These polymers also show significant aging effects, which are manifested in the permeability decline over time.

Another group of polymers which attract much interest for gas separation application are perfluoropolymers. Although poly(tetrafluoroethylene) (PTFE) used in many commercial applications shows very low gas permeability due to its semicrystalline nature, the new group of amorphous glassy perfluoropolymers (Teflon AF, Hyflon AD, Cytop) exhibit very high gas permeability. This is related to their high barrier to rotation between dioxole rings coupled with very low cohesive energy density (CED), which is typical for fluoropolymers. The potential use for this class of polymers is $\mathrm{N}_{2} / \mathrm{CH}_{4}$ separation due to their high light gas solubility relative to hydrocarbon solubility.

Polymers with intrinsic microporosity (PIM) represent a new class of high free volume polymers. Their structure is that of ladder polymers with a backbone of fused rings, which prevents large scale conformational change. Unlike typical ladder polymers, however, PIMs 
are soluble, membrane forming materials with good mechanical properties. This is due to the spiro-centre incorporated into their structure, which acts as a site of contortion preventing effective chains packing in the solid state. The characteristic feature of these polymers is their exceptionally high inner microporosity (SBET is around $800 \mathrm{~m}^{2} / \mathrm{g}$ for PIM-1) as well as high gas and vapour solubility, which is higher than that reported for any studied polymer including PTMSP. The most studied member of this family, PIM-1, makes the upper bound material for several gas pairs. Comparing to TR polymers, however, PIM materials show lower selectivities at similar prmeabilities for permanent gas separations. PIMs also undergo some degree of physical aging and plasticization and show less variations in permeability and selectivity resulting from chemical structure modifications than TR polymers.

Another group of gas separation materials includes rubbery materials such as polyethers or polyphosphazenes. Those materials have been identified as outstanding $\mathrm{CO}_{2}$ selective membrane materials. Generally, rubbery polymers exhibit high gas diffusion rates with low diffusivity selectivity. For those materials, improved separation ability while maintaining high permeation rate can only be achieved by preferential solubility in the polymer of the one permeant over the other species. This kind of separation usually involves acidic gases or organic vapours, which show strong interaction with some polar groups in the polymer chains. The innovative poly(ethylene oxide) based membrane materials able to selectively and efficiently separate $\mathrm{CO}_{2}$ from other gases have been already demonstrated. The field of polymeric gas separation membranes for a sustainable future has been recently reviewed through several articles, e.g. in [2-4]

\section{Inorganic membranes}

The limited thermal and chemical stability of polymeric membranes and upper bound restrictions has resulted in an increasing interest in inorganic membranes. These membranes are not bound to the trade-off limitations due to the fact that the size of the pores is fixed and true molecular sieving is possible. Here, the attention will be focused on a promising broad class of materials called tailored porous materials [5]. This would include zeolitic materials, metal organic frameworks (MOF), and carbon molecular sieves (CMS).
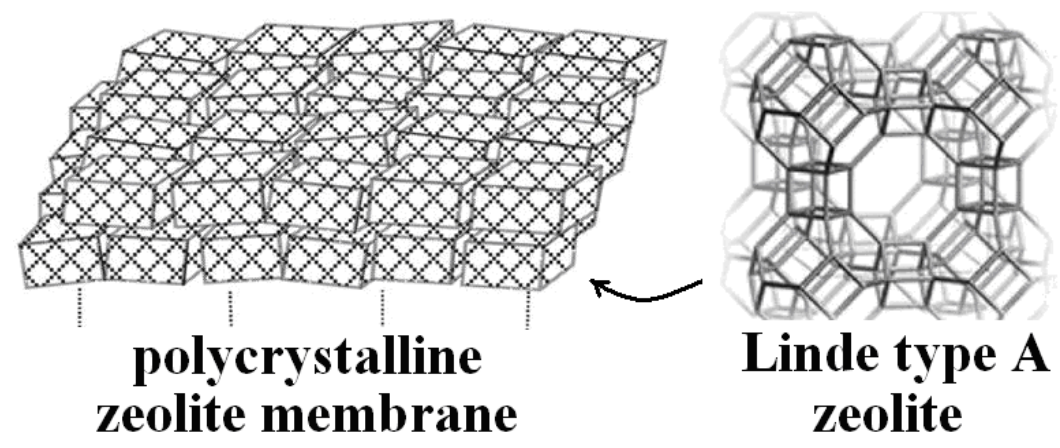

Fig.3 Scheme of a zeolite membrane microstructure.

Zeolites seem to be an ideal membrane material since they have uniform, molecular size pore (usually in the range of $0.3-1.3 \mathrm{~nm}$ ) and excellent thermal stability. The crystallographic 
structure determining the pore size along with chemical composition, which controls affinity influence the separation properties of a given zeolitic material. Generally, zeolitic membranes (Fig.3) show excellent separation performance for mixture with a strongly adsorbing component. Separation of gas mixture with negligible adsorption affinity with zeolite pore or separation at high temperatures when adsorption effect diminishes, i.e. when separation is controlled by diffusivity selectivity is less effective. This is due to the fact that even high quality zeolite membranes may contain microporous intercrystalline gaps or non-zeolitic pores, which dramatically degrade membrane separation properties. Thus, the potential application of the zeolite membranes mainly concerns natural gas purification. All silica DD3R membranes have been reported as highly $\mathrm{CO}_{2}$ selective and permeable for this application.

Metal organic frameworks (MOFs) are a relatively new class of microporous materials. They are composed of transition metals and transition metal oxides connected by organic linkages creating one-, two- or three dimensional microporous structures. MOFs cover a much wider pore size range than zeolites and present a high topological richness. Great diversity in pore size, shape and structure, possibility of further functionalization make them very interesting as membrane materials. Their extremely high porosity (much higher than that of zeolites) will create high fluxes, while the wide range of pore sizes, up to mesopore size range, would allow for various separations, including those of larger molecular isomers. The first MOF membrane from the ZIF family, ZIF-8 on a tubular support, showing outstanding $\mathrm{CO}_{2}$ fluxes has already been reported. In terms of selectivity, MOF membranes still do not appear to meet the expectations coming from their molecular sieving ability. This may be due to the flexibility of their structure deteriorating intrinsic molecular sieving and leading to the lower separation factors. Thus, the potential of MOF membranes has not been fully identified yet.

Carbon molecular sieves (CMS) are produced by pyrolyzing polymers. With respect to crystalline molecular sieves or MOFs, the pore size of CMS is not determined by the lattice constrains of the crystalline material. Therefore, a wider pore size distribution is usually characteristic for those amorphous materials. However, the breakthrough has been observed recently thanks to new oxidation techniques, which allow the pore size of CMS to be tuned more effectively. Several studies report now the selectivities and permeabilities for CMSs exceeding those exhibited by polymeric materials even for separation of gases showing small difference in their size like $\mathrm{CO}_{2} / \mathrm{N}_{2}$ or $\mathrm{O}_{2} / \mathrm{N}_{2}$. The structure and the performance of CMS membranes can be tailored by changing the nature of the precursor polymer as well as pyrolysis parameters. This structure consists of slit-like cavities of 1-2 nm size (micropores) connected by pore restrictions (ultramicropores) of smaller size able to discriminate between molecules based on their size. The brittleness of CMSs is a major drawback that hinders their commercialization.

\section{Mixed matrix membranes}

Mixed matrix membranes (MMM) are composed of inorganic particles embedded in a polymer matrix. This concept is thought to add better transport properties and higher thermal and chemical stability to the polymer phase avoiding some problems raised by pure inorganic membranes. Schematic representation of MMM is given in Fig. 4.

Generally, MMMs comprise inorganic porous particles such as zeolites, MOFs, CMSs or nonporous (impermeable) ones such as fumed silica, titanium dioxide or magnesium oxide. Depending on a particle type, different separation mechanisms are envisaged. The effect of a porous filler on the MMM performance is related to the pore size and its adsorption ability, if no defects are created in the polymer-particle interface. Those fillers when acting as molecular sieves can separate molecules based on their size and shape. The mechanism by 
which non-porous fillers influence the MMM transport properties is different. The presence of such particles disrupts chain packing and creates microvoids between two phases. The enhanced free volume in the resulting membrane usually reduces the diffusivity selectivity and makes the solubility selectivity the dominating factor for the overall separation process. Although the MMM concept seems very promising, the final success depends greatly on a suitable combinations of polymers and particles as well as on solving problems connected with polymer-filler compatibility, which should allow defect free membranes with controlled transport properties to be prepared. On the other hand, the optimization of MMM properties requires better understanding of the separation mechanisms occuring in these hybrid materials, particularly with respect to the phenomena taking place at the polymer-particle interface.

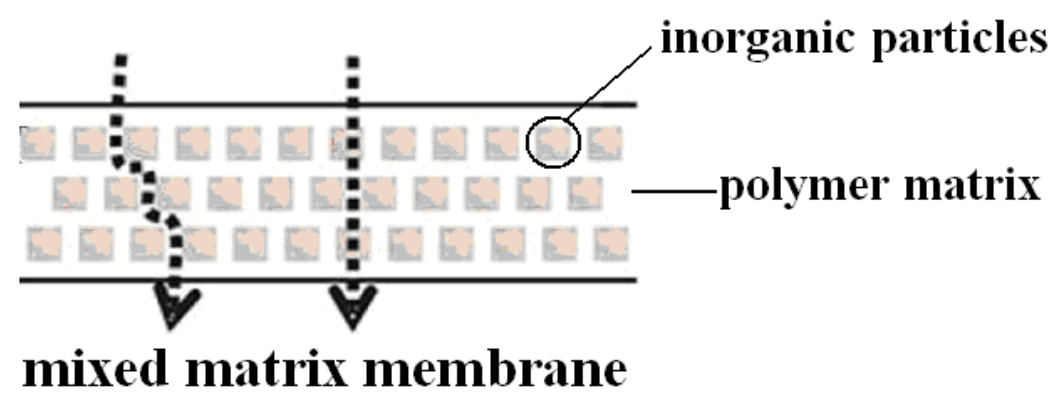

Fig.4 Schematic representation of a mixed matrix membrane (MMM).

\section{LITERATURE}

[1]. Robeson L.M., Correlation of separation factor versus permeability for polymeric membranes, J. Membrane Sci. 62 (1991) 165-185.

[2]. Yamploskii Y., Polymeric gas separation membranes, Macromolecules, 45 (2012) 32983311.

[3] Sanders D.F., Smith Z.P. Guo R., Robeson L.M., McGrath J.E., Paul D.R., Freeman B.D., Energy-efficient polymeric gas separation membranes for a sustainable future: A review, Polymer, 54 (2013) 4729-4761.

[4]. Lau C.H., Li P., Li F., Chung T-S., Paul D.R., Reverse-selective polymeric membranes for gas separations, Prog. Polym.Sci.38, (2013) 740-766.

[5]. Gascon J, Kapteijn F., Zornoza B., Sebastian V., Casado C., Coronas J., Practical Approach to Zeolitic Membranes and Coatings: State of the Art, Opportunities, Barriers, and Future Perspectives, Chem. Mater., 24, (2012) 2829-2844. 